\title{
INHIBITION OF CRYPTOCOCCUS NEOFORMANS BY PSEUDOMONAS AERUGINOSA
}

\author{
C. H. Teoh-Chan, P. Y. Chau, M. H. Ng and Pui Ching Wong \\ Department of Microbiology, University of Hong Kong, Hong Kong
}

\section{Plates IV AND V}

\begin{abstract}
DURING an investigation of Cryptococcus neoformans isolated from clinical specimens, we observed that growth of the fungus was considerably reduced in the vicinity of colonies of Pseudomonas aeruginosa. This phenomenon was first noted by Fisher (1954), who found that the inhibitory effect was exhibited only by living cultures of certain strains of $P$. aeruginosa. His efforts to isolate the inhibitor were unsuccessful and no further report on this phenomenon has since appeared. We report here a study of factors affecting the production of inhibitor by $P$. aeruginosa and an initial attempt to isolate and characterise the substances involved.
\end{abstract}

\section{MATERIALS AND METHODS}

Strains tested. Of 44 strains of $\boldsymbol{P}$. aeruginosa tested for their inhibitory activity on C. neoformans, 11 (nos. 8, 10, 17, 26, 39, 52, 10/55, 8/39, 283, 577 and 584) were obtained from Dr M. T. Parker, Colindale, England; 13 (Gillies and Govan's type strains 1, 2, 3, 4, $5,6,7,8$ and A, B, C, D, E) were obtained from the Public Health Laboratory, Edmonton, Canada; and 20 were freshly isolated from clinical specimens of wound and burn infections in Hong Kong. Fourteen strains of $C$. neoformans were tested for their sensitivity to the inhibitory effect of $P$. aeruginosa. These included 13 strains isolated from the cerebrospinal fluid of cryptococcal meningitis cases in Hong Kong and one strain, no. 1499.22, obtained from Dr Margarita Silva, Columbia University, NY, USA.

Media. The media used for this study were: Sabouraud Dextrose Agar (Oxoid), Tryptone Soya Agar (TSA, Oxoid), Diagnostic Sensitivity Test Agar (DST, Oxoid), and King's media A (without $\mathrm{CuCl}_{2}$ ) and $\mathrm{B}$. The latter two media were prepared according to the published formulae (King, Ward and Raney, 1954). Glucose was added to TSA, DST and the two King's media to final concentrations of $0.2,0.5,1 \cdot 0,2 \cdot 0$ and $3.0 \%$ to study its effect on the production of inhibitors.

Methods for testing the inhibitory action of $P$. aeruginosa on the growth of $C$. neoformans. In preliminary studies, two methods were used to demonstrate the inhibitory effect. (1) By means of the technique described by Fisher (1954), plates of TSA medium were initially lawned with $C$. neoformans, and after drying were inoculated centrally with $P$. aeruginosa (lower row, fig. 1). Small zones of inhibition were obtained. (2) With a method based on that described by Gillies and Govan (1966), P. aeruginosa from a 24-h broth culture was inoculated centrally in a circle of 1-cm diameter on TSA plates and incubated for 2 days. Growth was then removed, the residual cells were killed with chloroform vapour, and the surface of the plate was lawned with $C$. neoformans (top row, fig. 1). Zones of inhibition were larger, and it was apparent that inhibition did not depend on the presence of living $P$. aeruginosa.

Received 26 Feb. 1974; accepted 21 May 1974.

J. MED. MICROBIOL.-VOL. 8 (1975) 
For routine testing, another adaptation of the method of Gillies and Govan (1966) was used. Plates were inoculated with a central streak of $P$. aeruginosa. After overnight incubation, growth was removed by scraping with a slide and residual organisms were killed by exposure to chloroform vapour. Broth cultures of test strains of $C$. neoformans were streaked at right angles to the original linear inoculum of Pseudomonas and the results read after incubation for 3 days at $37^{\circ} \mathrm{C}$. Measurements represent widths in $\mathrm{cm}$ of the zones of complete inhibition (fig. 2).

Isolation and thin-layer chromatography of the inhibitory substance or substances. To facilitate the separation of $\boldsymbol{P}$. aeruginosa from extracellular substances, the organism was grown for $48 \mathrm{~h}$ on cellulose-acetate membranes placed on the surface of DST agar. The membranes were removed and discarded and the agar medium was homogenised and extracted repeatedly with chloroform. The pooled chloroform phases were in turn extracted with $0 \cdot 1 \mathrm{~N} \mathrm{HCl}$. The resulting pooled acidic aqueous phase contained mainly pyocyanin. The acid-extracted chloroform phase was concentrated by evaporation and analysed by ascending chromatography on activated kieselguhr; the solvent used was 7 parts of n-propanol and 3 parts of $17 \% \mathrm{NH}_{4} \mathrm{OH}$. The spots were viewed under ultraviolet (UV) light $(275 \mathrm{~nm})$ and their inhibitory activity assayed by separating the spots and eluting in chloroform. The eluates were concentrated by evaporation and absorbed on to paper discs, which were then dried and placed on the surface of plates seeded with the test strains of $C$. neoformans. Zones of inhibition were read after incubation for 3 days at $37^{\circ} \mathrm{C}$.

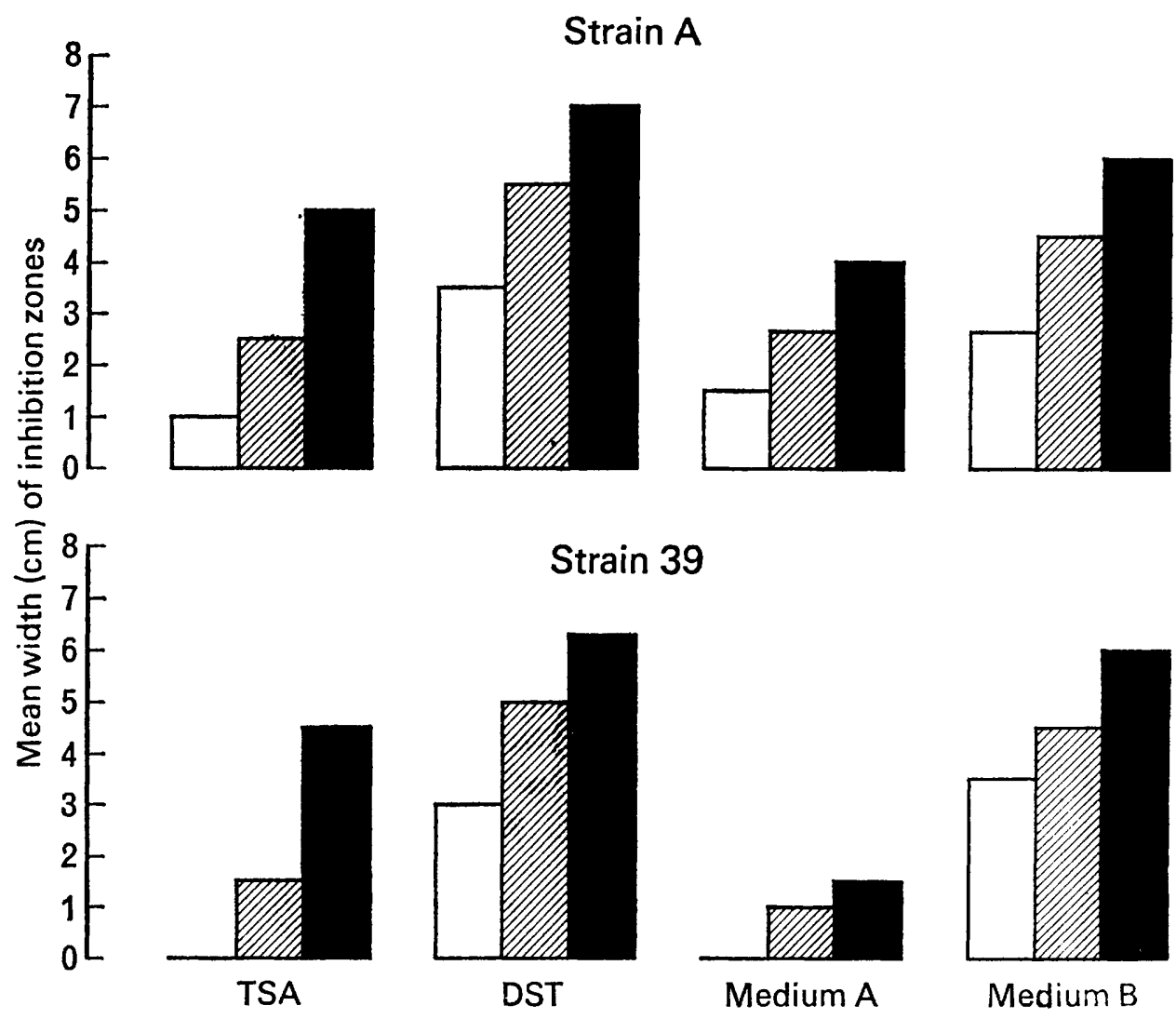

FIG. 3.-Effects of culture medium and time of incubation on the width of the zone of inhibition of C. neoformans (14 strains) by $P$. aeruginosa, strains A and $39, \square$ at $24 \mathrm{~h}, \mathbb{Z}$ at $48 \mathrm{~h}$, and $\mathrm{at} 72 \mathrm{~h}$. 


\section{INHIBITION OF CRYPTOCOCCUS BY PSEUDOMONAS}

\section{Glucose concentration}
$0.2 \%$
$0.5 \%$
$1.0 \%$
$2 \cdot 0 \%$
$3.0 \%$

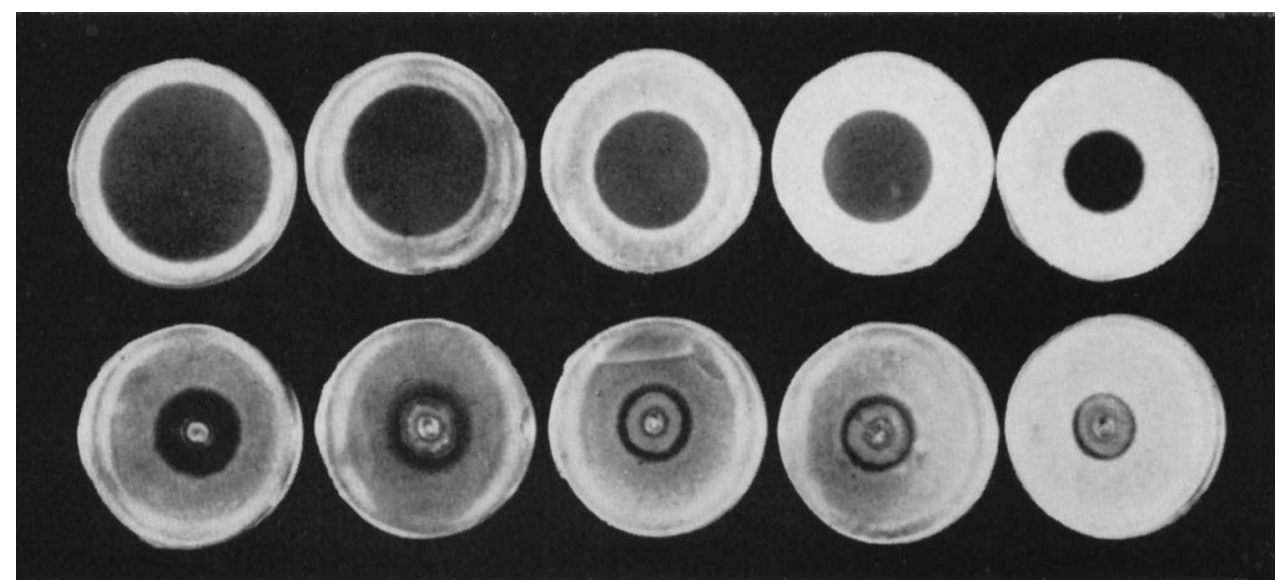

FIG. 1.-Inhibitory activity of Pseudomonas aeruginosa on Cryptococcus neoformans on plates containing various concentrations of glucose after incubation for 3 days at $37^{\circ} \mathrm{C}$. Upper row: DST plates previously inoculated at the centre with $P$. aeruginosa and incubated for 2 days. Surface lawned with $C$. neoformans after removal of viable Pseudomonas. Lower row: TSA plates initially lawned with $C$. neoformans. After drying, the same inocula of Pseudomonas were inoculated at the centres.

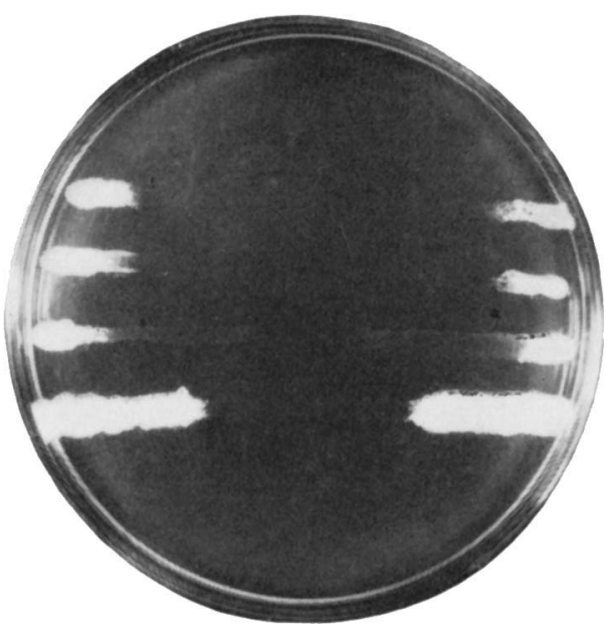

FIG. 2.-Complete and partial inhibition of $C$. neoformans by $P$. aeruginosa. 


\section{InHIBITION OF CRYPTOCOCCUS BY PSEUDOMONAS}
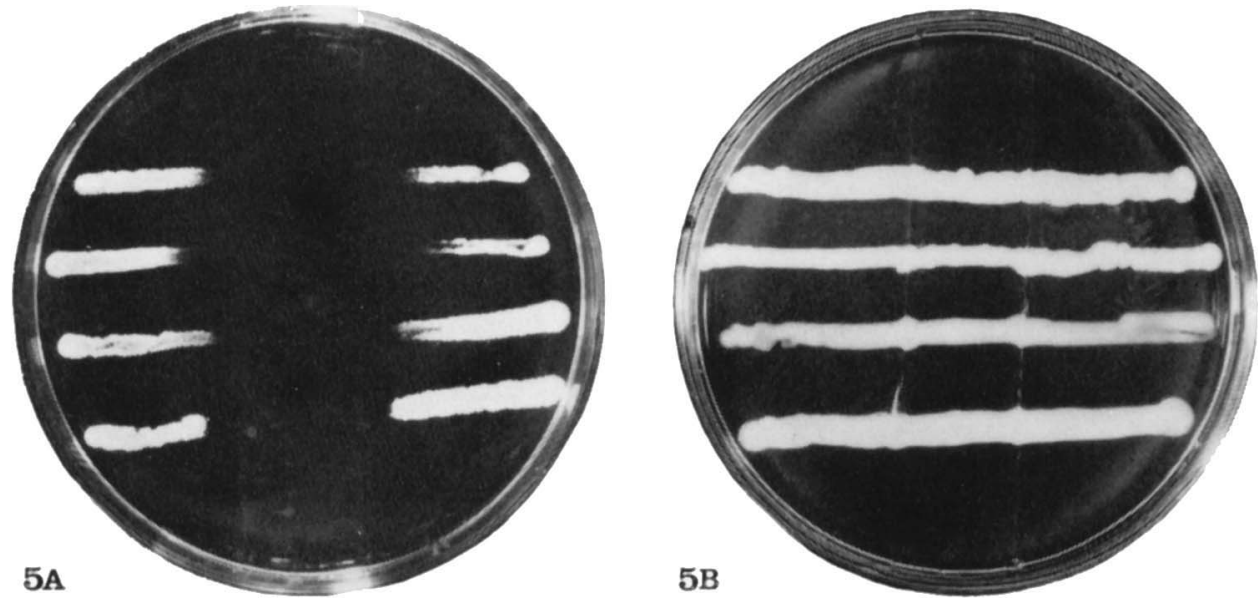

FIG. 5.-Effect of glucose on the production of inhibitory substances by $P$. aeruginosa: A-P. aeruginosa grown on TSA medium without glucose; B-the same culture grown on TSA medium containing $3 \%$ glucose. 


\section{RESULTS}

All $44 P$. aeruginosa strains inhibited the $14 C$. neoformans strains tested, but the degree of inhibition varied with the strains used, the time of incubation, the type of medium and its glucose content.

Inhibitory activity of $P$. aeruginosa strains. In general, the 20 freshly isolated Pseudomonas strains showed greater inhibitory activity than the 24 standard strains. Among the 24 standard Pseudomonas strains tested, two groups could be distinguished by their respective abilities to produce inhibitor substance; in one group, as represented by Gillies and Govan's strain A, inhibitory substance could invariably be detected within the first $24 \mathrm{~h}$ of incubation regardless of the medium used. In contrast, production of inhibitory substance within the second group, as represented by strain 39 varied according to the medium used and the time of incubation (fig. 3).

Sensitivity of C. neoformans. All the $14 C$. neoformans strains were sensitive to the inhibitory substances produced by $P$. aeruginosa. The more mucoid isolates (eight of the 14 strains) appeared to be the more sensitive. The less
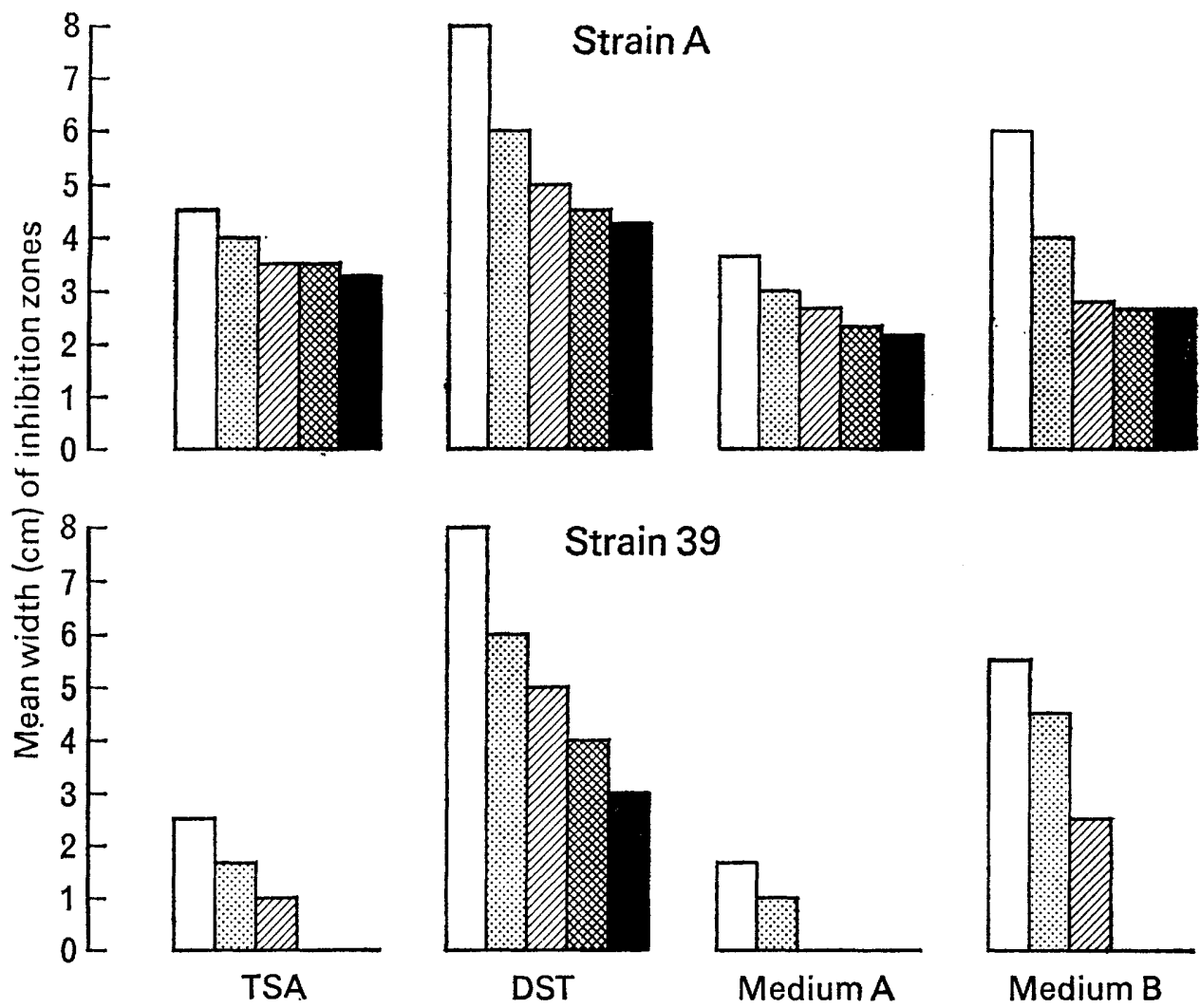

Frg. 4.-Effect of adding glucose to various media on the width of the zone of inhibition of C. neoformans (14 strains) by $P$. aeruginosa, strains $A$ and 39 , with a glucose concentration of $0.2 \% \square, 0.5 \%$ 圈, $1.0 \%, 2.0 \%$ 国, $3.0 . \%$ m. 
sensitive strains sometimes showed partial inhibition with delayed and sparse growth (fig. 2). Agar blocks removed from the centre of complete inhibition zones and inoculated into $50 \mathrm{ml}$ of Dextrose Broth (Oxoid) showed no growth of $C$. neoformans in daily subcultures incubated at $37^{\circ} \mathrm{C}$ for 7 days. This indicates that the inhibitor or inhibitors derived from $P$. aeruginosa appeared to be fungicidal against $C$. neoformans.

Effect of culture medium and incubation time. The size of the inhibition zone increased with the length of the primary incubation period. The widest zones of inhibition were obtained on DST Agar and King's medium B followed in order by TSA medium and King's medium A, whereas on Sabouraud Dextrose Agar, the results obtained were irregular. Fig. 3 shows the results obtained with two representative strains of $P$. aeruginosa.

Effect of glucose concentration. The addition of glucose to each of the four media reduced the inhibitory effect of all Pseudomonas strains (figs. 1 and 4). Of the four media used, incorporation of glucose affected inhibition least on DST agar.

To determine whether the decrease of inhibitory activity with increasing glucose concentration was due to increased resistance of $C$. neoformans, $P$. aeruginosa strain 39 was spread on the surfaces of two TSA plates one with and one without $3.0 \%$ glucose. After incubation for $48 \mathrm{~h}$ at $37^{\circ} \mathrm{C}$, the growth was removed and the plates were exposed to chloroform vapour. The agar medium in both plates was then cut away leaving a $2 \mathrm{~cm}$ wide strip in the middle. The discarded agar was replaced with freshly melted TSA medium, but the fresh agar contained $3.0 \%$ glucose for the plate in which the original strip contained none, and no glucose for the plate in which the central strip contained $3.0 \%$ glucose. After solidification, broth cultures of $C$. neoformans were streaked at right angles to the central strips and the result read after incubation for 3 days at $37^{\circ} \mathrm{C}$ (fig. 5). The growth of $C$. neoformans was completely inhibited over the strip without glucose and in a zone that extended into the newly added TSA medium with $3.0 \%$ glucose. On the plate in which the central strip contained glucose no inhibition occurred.

Inhibitory properties of the acid-insoluble, chloroform-extractable material. Up to five UV-absorbing spots were detected in the concentrate of the acidextracted chloroform phase, none of which migrated with the same $\mathrm{Rf}$ value as that of pyocyanin (0.62). Inhibitory activity against $C$. neoformans was found to be associated with only one of the spots, which had an RF value of $0 \cdot 77$. Recovery of inhibitory activity judged by the size of the inhibitory zone was poor compared with that of the chloroform concentrate before chromatography.

\section{Discussion}

The literature about the inhibitory effect of $P$. aeruginosa on $C$. neoformans is scanty and equivocal. Fisher (1954) first noticed that the growth of $C$. neoformans was inhibited by $P$. aeruginosa, but only by certain living strains. Various attempts to isolate the inhibitor were unsuccessful. Fedors (1959) extracted broth cultures of $P$. aeruginosa, and found that both chloroform 
extracts containing $\alpha$-oxyphenazine and ether extracts of the chloroformextracted residue inhibited the growth of Histoplasma capsulatum and Blastomyces dermatitidis, but failed to inhibit the growth of C. neoformans.

The present experiments show that the presence of glucose in the medium interferes with the production of the Pseudomonas inhibitor but not with its activity, and this probably accounts for the irregular results obtained by Fisher (1954) who used Sabouraud's agar containing 3\% glucose. Furthermore, he cultured both organisms simultaneously, thus limiting the time available for production of the inhibitor.

Although both of these authors demonstrated that the pigment pyocyanin was not inhibitory to $C$. neoformans, $H$. capsulatum or $B$. dermatitidis, Mangan (1969) showed that pyocyanin inhibited the germination of spores of Aspergillus terreus. Our results agree with those of Fisher (1954) and Fedors (1959) in showing that the inhibitory activity of $P$. aeruginosa against $C$. neoformans is not related to pyocyanin, because least inhibitory activity was demonstrated on the pyocyanin-enhancing King's medium A, and chromatographic analysis clearly separated the inhibitor from pyocyanin. Because recovery in the chromatographic separation was poor, however, it was uncertain whether the substance with an $\mathrm{Rf}$ value of 0.77 was the sole inhibitor in the crude extract, and its chemical nature was not elucidated.

\section{SUMMARY}

Pseudomonas aeruginosa was found to produce a factor or factors that inhibited Cryptococcus neoformans and appeared to be extracellular because the anti-C. neoformans activity was readily demonstrable in medium after the removal and killing of Pseudomonas organisms. Production of the inhibitor material was greatest in DST Agar after prolonged incubation and was reduced in the presence of glucose. A part of the inhibitory material was found to be chromatographically distinct from pyocyanin.

\section{REFERENCES}

Fedors, P. 1959. Inhibition of Histoplasma capsulatum and Blastomyces dermatitidis by Pseudomonas aeruginosa in vitro. Mycopathologia, 11, 128.

FisHer, A. M. 1954. Inhibition of growth of Cryptococcus neoformans by cultures of Pseudomonas aeruginosa. Bull. Johns Hopkins Hosp., 95, 157.

Gillies, R. R. AND Govan, J. R. W. 1966. Typing of Pseudomonas pyocyanea by pyocine production. J. Path. Bact., $91,339$.

KING, E. O., WARD, M. K. AND RANEY, D. E. 1954. Two simple media for the demonstration of pyocyanin and fluorescin. J. Lab. clin. Med., 44, 301.

MANGAN, A. 1969. Interactions between some aural Aspergillus species and bacteria. J. gen Microbiol., 58, 261. 